\title{
Polymer Chain Dynamics. II. Intermittent Process of Orientational Relaxation of Polystyrene Main Chain
}

\author{
Fumiaki Tsunomori, Hideharu UsHIKI, and Kazuyuki HorIE* \\ Graduate School of Bio-Applications and Systems Engineering, Tokyo University of \\ Agriculture and Technology, Fuchu, Tokyo 183, Japan \\ * Faculty of Engineering, The University of Tokyo, Hongo, Bunkyo-ku, Tokyo 113, Japan
}

(Received November 16, 1995)

\begin{abstract}
Polystyrene chains having an anthryl group in the middle of its back bone (PSAPS), whose degrees of polymerization are 3200,2500 , and 730 , were used to detect orientational relaxation of polymer segments. In order to explain the power law behavior of fluorescence anisotropy decay curves of PSAPS chain, it was assumed that the orientational relaxation of segments is caused by the reaction between segments and solvent molecules. Further the distribution of interval time between relaxation phenomena was considered. According to above two assumptions, a hyperbolic function $r(t) \sim r_{0}\left(t / \tau_{\text {eff }}\right)^{-\beta}$ have been derived as an orientational auto-correlation function. We propose the "intermittent process" of polymer segments as one of the probable description for the orientational relaxation process of polystyrene back bone. The hyperbolic exponent $\beta$ is related to the characteristic activation energy $E_{\mathrm{a}}$ of a polymer segment. The value of $E_{\mathrm{a}}=7 \mathrm{~kJ} \mathrm{~mol}^{-1}$ for polystyrene chain evaluated by $\beta$ is in good agreement with hitherto reported values. Effective relaxation time $\tau_{\text {eff }}$ corresponds to the minimum reaction radii $\xi_{0}$ between a segment and a solvent molecule, i.e., the diffusivity of solvent molecules. Temperature dependence of $\xi_{0}$ is consistent with that of the reciprocal of solvent viscosity.

KEY WORDS Intermittent Process / Polystyrene Main Chain / Hyperbolic Function / Fluorescence Depolarization / Long Time Tail /
\end{abstract}

Diffusive motion is the most important elemental process in the self-organization phenomenon. Diffusion refers to the transport process of matter in space with time. The study of the self-organization phenomenon is equivalent to that of relationship between space and time. We have been claiming the significance of a hierarchical viewpoint for the vast number of motion. ${ }^{1-3}$. The polymer system has almost motion correlating the self-organization process. Thus it is necessary to establish first appropriate methods to estimate each motion for understanding the self-organization phenomenon.

As considering primary process of the conformational change of a polymer chain, the orientational relaxation motion is firstly mentioned as an elemental motion in a microscopic region. In the last twenty years, many theoretical and experimental studies of orientational relaxation phenomena of a polymer chain were reported by many authors. ${ }^{4-10}$ Most of studies assumed that orientational relxation of polymer segments consists of a few modes. However, in reality, a polymer chain has the vast number of internal freedom about motion. It is important to make a realistic picture of orientational relaxation behavior on the basis of a model reflecting the distribution of relaxation time.

The trend in experimental studies on the orientational relaxation process of a segment at the center of a polymer back bone began with the Valeur's reports. ${ }^{4,11,12}$ They studied an orientational auto-correlation function describing fluorescence anisotropy decay curves of polystyrene chain having an anthryl group in the middle of its back bone (PSAPS) in dilute solution. The VJGM function was proposed,

$$
r(t) \sim \exp \left(-t / \tau_{2}\right) \exp \left(t / \tau_{1}\right) \operatorname{erfc}\left(\sqrt{t / \tau_{1}}\right)
$$

on the basis of a realistic description of the relaxation called by the 3 -bond motion, where $\tau_{1}$ and $\tau_{2}$ are relaxation times corresponding to the conformational jump and the out-of-lattice motions. They suggested that an orientational auto-correlation function is to be $r(t) \sim t^{-1 / 2}$ in the later stage of the relaxation process. That is to say, they found the fluorescence anisotropy of PSAPS obeys the power law at long times.

Hall and Helfand ${ }^{7}$ and Viovy, Monnerie, and Brochon ${ }^{8}$ respectively proposed the $\mathrm{HH}$ function

$$
r(t) \sim \exp \left(-t / \tau_{2}\right) \exp \left(-t / \tau_{1}\right) I_{0}\left(t / \tau_{1}\right)
$$

and the GDL function

$$
r(t) \sim \exp \left(-t / \tau_{2}\right) \exp \left(-t / \tau_{1}\right)\left(I_{0}\left(t / \tau_{1}\right)+I_{1}\left(t / \tau_{1}\right)\right)
$$

where $\tau_{1}$ and $\tau_{2}$ are relaxation times corresponding the correlated and isolated jump motions of polymer segments. However these expressions are correct only at the short-time region.

Bendler and Yaris $^{6}$ proposed the BY function consisting of a long-wavelength and a short-wavelength cutoff in the continuous-mode spectrum of

$$
\frac{\mathrm{d} P}{\mathrm{~d} t}=W \frac{\mathrm{d}^{2} P}{\mathrm{~d} x^{2}}
$$

where $P$ and $x$ are the probability density of relaxation and a certain distance along the chain. The BY function taking into account the vast number of relaxation modes has the function form as

$$
\begin{aligned}
r(t) \sim 1 / 2 \sqrt{\pi} & \left(\operatorname{erfc}\left(\sqrt{t / \tau_{1}}\right)\right. \\
& \left.-\operatorname{erfc}\left(\sqrt{t / \tau_{2}}\right)\right) /\left(\sqrt{t / \tau_{2}}-\sqrt{t / \tau_{1}}\right)
\end{aligned}
$$

where $\tau_{1}$ and $\tau_{2}$ indicate the arbitrary cutoff frequencies. It is difficult to relate these parameters to realistic molecular motions. It is worthwhile, however, to note that the BY function considers the distribution of relaxation time of polymer segments.

On the other hand, non-exponential relaxation be- 
haviors of amorphous materials have been studying with two kinds of empirical decay functions, ${ }^{13,14}$

$$
f(t) \sim \exp \left(-\left(\frac{t}{\tau}\right)^{\beta}\right)
$$

and

$$
f(t) \sim t^{-\beta}
$$

The former is called Kohlrausch-Williams-Watts function (or stretched exponential function) $)^{15,16}$ and the latter is called a hyperbolic function (or power law decay function). Behaviors of these functions have been interpreted from two viewpoints. The first is the distribution of relaxation times, ${ }^{13,17}$ the second is the mode coupling theory. ${ }^{18,19}$ Recently, many studies support the viewpoint of the distribution of relaxation rate. ${ }^{20-25}$

In our preceding paper, ${ }^{26}$ we found out that fluorescence anisotropy decay curves of a polystyrene main chain are successfully reproducible with a hyperbolic function. In this paper, we discuss the primary process of orientational relaxation of polymer segments on the basis of fluorescence anisotropy decay curves.

\section{EXPERIMENTAL}

Polystyrenes having an anthryl group in the center of its back bone (PSAPS) were synthesized in the same manner as reported previously. ${ }^{27}$ PSAPS chain was given by the direct attack of living polystyryl monoanions, which were polymerized anionically in tetrahydrofuran with cumyl potassium as the initiator under a high vacuum system, with stoichiometric amount of 9,10-bis(chloromethyl)anthracene. Chemical structure of PSAPS is shown in Figure 1. Table I shows the values of the number-average molecular weight $M_{n}$ and the molecularweight distribution $M_{w} / M_{n}$ measured with a gel permeation chromatography (Toyo Soda HLC-802UR), as well as the percentages of anthryl group in the polymers measured with an ultraviolet spectrophotometer (Shimadzu MPS-5000).

The concentration of PSAPS dissolved in a mixed solvent of tripropionin (glyceryl tripropionate) and ethyl acetate was kept less than $0.1 \mathrm{wt} \%$ at the final step of preparation. The volume fraction of tripropionin in the

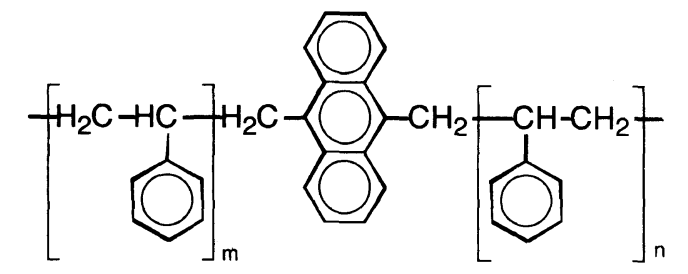

Figure 1. Chemical structure of PSAPS chain.

Table I. Characterization of PSAPS chain

\begin{tabular}{crccc}
\hline Sample & $D P^{\mathrm{a}}$ & $M_{n}$ & $M_{w} / M_{n}$ & Dye contents \\
\hline PSAPS3200 & 3200 & $3.3 \times 10^{5}$ & 1.39 & 95 \\
PSAPS2500 & 2500 & $2.8 \times 10^{5}$ & 1.82 & 100 \\
PSAPS730 & 730 & $7.6 \times 10^{4}$ & 2.40 & 86 \\
\hline
\end{tabular}

${ }^{a}$ Degree of polymerization. mixed solvent was 0.94. Sample solutions were degassed by several freeze-pump-thaw cycles, and were enclosed in a Pyrex tube cell.

\section{Measurement}

The absorption, excitation, emission, and depolarization spectra corrected by the concentrated solution of rhodamine B to compensate the spectroscopic sensitivity of optical equipments, and time-resolved emission decay curves of PSAPS were measured by the PLASMA system. ${ }^{3,26}$ The excitation and emission wavelengths were 384 and $430 \mathrm{~nm}$, respectively. Measurements were carried out at $20.0,25.0,30.0,37.5,40.0$, and $42.5^{\circ} \mathrm{C}$ with a thermostat.

Fluorescence decay curves $F_{\text {obs }}(t)$ and fluorescence anisotropy decay curves $r_{\text {obs }}(t)$ were calculated by

$$
F_{\text {obs }}(t)=I_{\mathrm{Vv}}(t)+2 G I_{\mathrm{VH}}(t)
$$

and

$$
r_{\mathrm{obs}}(t) \equiv \frac{I_{\mathrm{Vv}}(t)-G I_{\mathrm{VH}}(t)}{I_{\mathrm{Vv}}(t)+2 G I_{\mathrm{VH}}(t)}
$$

respectively. Where $G$ is the compensating factor for anisotropic sensitivity of optics in the apparatus (a timeresolved fluorophotometer, Horiba NAES-1100). $G$ is defined by

$$
G=\int I_{\mathrm{HV}}(t) \mathrm{d} t / \int I_{\mathrm{HH}}(t) \mathrm{d} t
$$

$I_{\mathrm{VV}}(t), I_{\mathrm{VH}}(t), I_{\mathrm{HH}}(t)$, and $I_{\mathrm{HV}}(t)$ denote polarized fluorescence intensity measured through a band path filter (Toshiba: KL43). Subscripts V and $\mathrm{H}$ represent the parallel and the perpendicular direction to the vertical line. The first suffix indicates the direction of an incident light and the second an emitted light.

\section{Calculation}

Fluorescence decay $F_{\text {cal }}(t)$ and fluorescence anisotropy decay $r_{\text {cal }}(t)$ are reproduced by

$$
F_{\text {cal }}(t) \sim \int_{0}^{t} P(T) S(t-T) \mathrm{d} T
$$

and

$$
r_{\mathrm{cat}}(t) \sim \frac{\int_{0}^{t} P(T) D(t-T) S(t-T) \mathrm{d} T}{\int_{0}^{t} P(T) S(t-T) \mathrm{d} T}
$$

respectively. Where $P(t), S(t)$, and $D(t)$ denote the response function of the apparatus, a fluorescence decay function and a true orientational auto-correlation function, respectively. We assumed that $P(t)$ is an exciting light pulse, and $S(t)$ is a double exponential function.

Deconvolution and curve fitting calculation were carried out on the basis of the Wahl's method ${ }^{28}$ and the quasi-Marquardt algorithm, ${ }^{29}$ respectively. The suitability of a function to data was evaluated by a value of the residual sum of squares, $\chi^{2}$. Adequate values of variable parameters of a trial function were computed until the variation of $\chi^{2}$ become less than $1 \times 10^{-6}$. On fitting data with $S(t), \chi^{2}$ was calculated by 


$$
\chi^{2}=\frac{1}{t_{2}-t_{1}-4} \int_{t_{1}}^{t_{2}} w(t)\left[F_{\text {obs }}(t)-F_{\text {cal }}(t)\right]^{2} \mathrm{~d} t
$$

where $w(t)$ is a weight function at time $t$, and $t_{1}$ and $t_{2}$ indicate the initial and the final times of the fitting range, respectively. The weight function ${ }^{28}$ adopted for $F_{\text {cal }}(t)$ is

$$
w(t)=\frac{1}{I_{\mathrm{Vv}}(t)+4 G^{2} I_{\mathrm{VH}}(t)}
$$

On evaluating parameters in $D(t)$, eq 13 was substituted by

$$
\chi^{2}=\frac{1}{t_{2}-t_{1}-m} \int_{t_{1}}^{t_{2}} w(t)\left[r_{\text {obs }}(t)-r_{\text {cal }}(t)\right]^{2} \mathrm{~d} t
$$

where $m$ is the number of variable parameters in the function $D(t)$. The weighting function was replaced by ${ }^{28}$

$$
w(t)=\frac{3 F_{\mathrm{obs}}(t)}{1+G+3 G r_{\mathrm{obs}}(t)-3 r_{\mathrm{obs}}(t)^{2}-2(2 G-1) r_{\mathrm{obs}}(t)^{3}}
$$

\section{RESULTS AND DISCUSSION}

\section{Fluorescence Anisotropy Decay Curves}

Fluorescence anisotropy decay curves of PSAPS measured at $30^{\circ} \mathrm{C}$ are shown in Figures 2, 3, and 4. The fluorescence anisotropy decreased with increasing time. At initial stage of this relaxation process, the fluorescence anisotropy decayed sharply, and it had a long time tail at final stage of the relaxation process. If a probe molecule can rotate isotropically, fluorescence anisotropy obeys the exponential law as $r(t) \sim \exp (-t / \tau)$. However decay curves suggest that a probe molecule incorporated in a back bone of a polymer chain can move in the wake of orientational relaxation motion of a polymer back bone.

\section{Hyperbolic Function as Orientational Auto-Correlation Function}

We pointed out that a hyperbolic function is useful to express a fluorescence anisotropy decay curve in the long time region. ${ }^{26}$ Hyperbolic function has the form

$$
r(t)=r_{0}\left(\frac{t}{\tau_{\text {eff }}}\right)^{-\beta}
$$

where $r_{0}, \tau_{\text {eff }}$, and $\beta$ are a constant, the effective orientational relaxation time and a hyperbolic exponent, respectively.

Let us consider that orientational relaxation of polymer segments consisting of a few monomers is caused by the reaction with solvent molecules migrating in a solution.

$$
A+B \rightarrow A^{\prime}+B^{\prime}
$$

where $A, B, A^{\prime}$ and $B^{\prime}$ are a polymer segment orienting to the initial direction, a solvent molecule before reaction, a polymer segment relaxed, and a solvent molecules after reaction. The number of $A$ as a function of time $t$ is given by

$$
\frac{\mathrm{d} n_{A}}{\mathrm{~d} t} \sim-R_{0} D n_{A} n_{B}
$$

where $n_{i}$ denotes the density of species $i, R_{0}$ the reaction

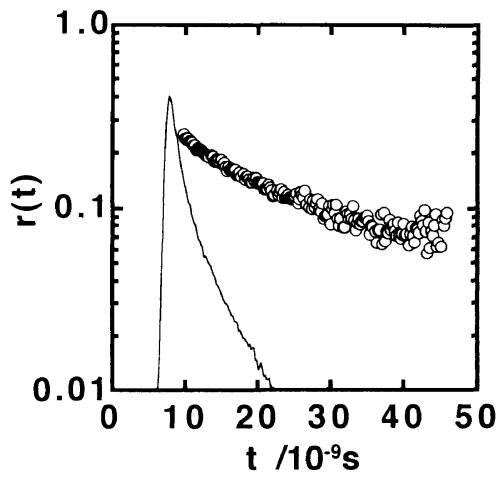

Figure 2. Fluorescence anisotropy decay curve of an anthryl group in PSAPS3200 chain measured at $30^{\circ} \mathrm{C}$.

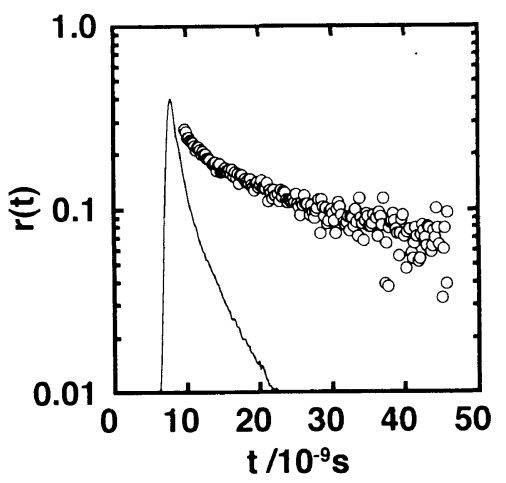

Figure 3. Fluorescence anisotropy decay curve of an anthryl group in PSAPS2500 chain measured at $30^{\circ} \mathrm{C}$.

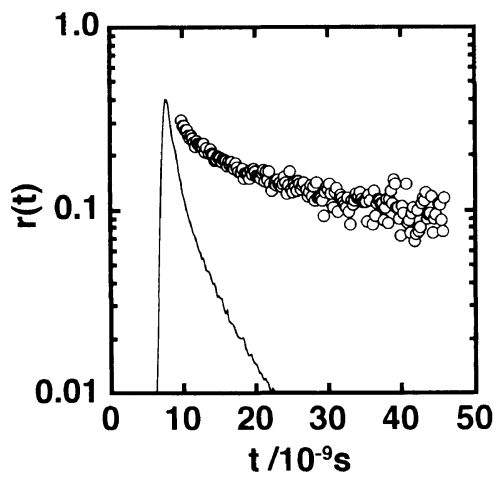

Figure 4. Fluorescence anisotropy decay curve of an anthryl group in PSAPS730 chain measured at $30^{\circ} \mathrm{C}$.

radii and $D$ the diffusion coefficient which is the sum of $D_{A}$ and $D_{B} \cdot n_{A}$ as a function of time is equivalent to the orientational auto-correlation function $r(t)$ of polymer segments,

$$
n_{A}(t)=r(t)
$$

Mean square distance of migration $\left\langle L^{2}(t)\right\rangle$ of $B$ is assumed to be given according to Tuac ${ }^{21}$

$$
\left\langle L^{2}(t)\right\rangle=D t
$$

We shall assume that the orientation of a polymer segment relaxes after the segment is activated by the reaction with solvent molecules above the threshold $E_{\mathrm{c}}$. In this case, the state density of polymer segments is written as

$$
\rho(E)=\rho_{\mathrm{c}} \exp \left(-\frac{E-E_{\mathrm{c}}}{k_{\mathrm{B}} T_{\mathrm{r}}}\right)
$$


where $E, \rho_{\mathrm{c}}$ and $k_{\mathrm{B}}$ are potential energy of a polymer segment, the state density at $E_{\mathrm{c}}$ and Boltzmann constant, respectively. $T_{\mathrm{r}}$ corresponds to the characteristic activation energy of polymer segments as

$$
E_{\mathrm{a}}=k_{\mathrm{B}} T_{\mathrm{r}}
$$

According to Orenstein, ${ }^{17}$ it is assumed that the interval time $t_{\mathrm{w}}$ for orientational relaxation of a segment at energy $E$ is expressed as

$$
\frac{1}{t_{\mathrm{w}}}=v_{\mathrm{ph}} \exp \left(-\frac{E-E_{\mathrm{c}}}{k_{\mathrm{B}}\left(T-T_{\mathrm{r}}\right)}\right)
$$

where $v_{\mathrm{ph}}$ and $T$ indicate the frequency of phonon and temperature, respectively. On rearranging eq 24 , we obtain

$$
-\frac{E-E_{\mathrm{c}}}{k_{\mathrm{B}}}=\left(T-T_{\mathrm{r}}\right) \ln \frac{1}{t_{\mathrm{w}} v_{\mathrm{ph}}}
$$

Equation 22 is rearranged as

$$
\rho(E)=E_{\mathrm{c}}\left(\frac{1}{t_{\mathrm{w}} v_{\mathrm{ph}}}\right)^{\left(T-T_{\mathrm{r}}\right) / T_{\mathrm{r}}}
$$

Here the distribution function $\Psi\left(t_{\mathrm{w}}\right)$ of interval times for relaxtion is related to the state density $\rho(E)$ as

$$
\rho(E) \mathrm{d} E \sim \Psi\left(t_{\mathrm{w}}\right) \mathrm{d} t_{\mathrm{w}}
$$

On integrating both members of eq 27 , and substitued eq 26 into above expression, we obtain

$$
\begin{aligned}
\Psi\left(t_{\mathrm{w}}\right) & \sim t_{\mathrm{w}}^{-1-\left(T-T_{\mathrm{r}}\right) / T_{\mathrm{r}}} \\
& \equiv t_{\mathrm{w}}^{-1-\gamma}
\end{aligned}
$$

When $\Psi\left(t_{\mathrm{w}}\right)$ is expressed by eq 28 , mean square distance of migration $\left\langle L^{2}(t)\right\rangle$ of a species $B$ is given ${ }^{13}$ by

$$
\left\langle L^{2}(t)\right\rangle \sim t^{\gamma}
$$

Substituting above equation into eq $21, D$ is expressed as a function of time,

$$
D \sim t^{\gamma-1}
$$

Therefore eq 19 is changed as

$$
\frac{\mathrm{d} n_{A}}{\mathrm{~d} t}=-\frac{\xi_{0}}{t^{1-\gamma}} n_{A} n_{B}
$$

where $\xi_{0}$ is the minimum radii of reaction between $A$ and $B$ at $T$.

Here we estimate the density of polymer segments and solvent molecules. Firstly, the density of polymer segments in a polymer sphere is evaluated by

$$
n_{A}(l)=N\left(\frac{3}{2 \pi R_{G}^{2}}\right)^{3 / 2} \exp \left(-\frac{3 l^{2}}{2 R_{G}^{2}}\right)
$$

where $l$ and $N$ are the distance from the center of gravity of its polymer sphere and the number of segment, respectively. $R_{G}^{2}$ is given by

$$
R_{G}^{2}=\frac{1}{3} \alpha^{2} N b^{2}
$$

where $\alpha$ is the expansion factor, and $b$ is the length of a segment. If one segment consists of two monomers, then $b=3.1 \times 10^{-8} \mathrm{~cm}$. The value of the expansion fac- tor $\alpha$ is $0.93 .{ }^{30}$ The numbers of segment are $N=1600$ for PSAPS3200, $N=1250$ for PSAPS2500 and $N=365$ for PSAPS730, respectively. According to eq 32, the segment densities for PSAPS chains are evaluated as $n_{A}(0)_{3200}=1.8 \times 10^{21} \mathrm{~cm}^{-3}, n_{A}(0)_{2500}=2.1 \times 10^{21} \mathrm{~cm}^{-3}$ and $n_{A}(0)_{730}=3.8 \times 10^{21} \mathrm{~cm}^{-3}$, respectively. On the other hand, the density of solvent molecules measured at $27^{\circ} \mathrm{C}$ was $n_{B}=2.5 \times 10^{21} \mathrm{~cm}^{-3}$. It can be presumed that the densities of polymer segments and solvent molecules are in the same order. Thus we should solve eq 31 under the condition of $n_{A} \simeq n_{B}$.

Solving eq 31 with the condition $n_{A} \simeq n_{B}$, one can obtain the $n_{A}$ as a function of time $t$,

$$
n_{A}(t)=\frac{n_{0}}{1+\frac{\xi_{0} n_{0}}{\gamma} t^{\gamma}}
$$

where $n_{0}$ is the density of $n_{A}$ at $t=0$. After a long time, the above equation is changed as

$$
n_{A}(t)=\frac{\gamma}{\xi_{0}} t^{-\gamma}
$$

We obtained two relations:

$$
\begin{gathered}
\beta=\gamma \\
\xi_{0} \sim \frac{\beta}{r_{0} \tau_{\text {eff }}^{\beta}}
\end{gathered}
$$

In this manner, we have obtained hyperbolic relaxation function of polymer segments as the orientational autocorrelation function.

Above result is equivalent to saying that the primary process of orientational relaxation of polymer segments is an "intermittent process". That is, orientational relaxation of a segment start and stop at intervals. Figure 5 shows the comparison of our idea with a hitherto one. According to the hitherto idea (Figure 5(a)), a segment oriented to veritcal at $t=t_{0}$ rotates smoothly with some velocity, and angle between initial and final $\left(t=t_{3}\right)$ direction reaches to some value $\theta$. With this model, the relaxation time given by an orientational auto-correlation function can be related to the velocity of rotational motion of a segment. On the other hand, the intermittent relaxation process (Figure 5(b)) suggests that a segment along to vertical at $t=t_{0}$ instantaneously changes at $t=t_{1}$

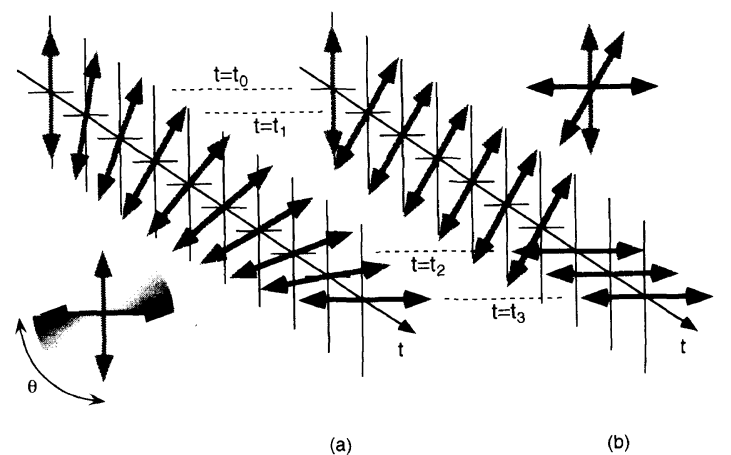

Figure 5. Schematic representation of (a) gradual process and (b) intermittent process of orientational relaxation of a polymer segment. In the case of (a), orientation of a segment gradually relaxes with time. On the other hand, in the case of (b), orientation of a segment instantaneously relaxes at $t=t_{2}$ and $t=t_{3}$. 
and $t=t_{2}$. Therefore relaxation time $\tau_{\text {eff }}$ does not refer to the rotational velocity of a segment. The value of $\tau_{\text {eff }}$ is associated with the minimum radii of reaction $\xi_{0}$ given by eq 37 .

\section{Comparison with Other Studies}

The intermittent process for the orientational relaxation of chain segments is not so strange picture. Because many phenomena comprised of primary processes which occur with the interval times have been reported as follows.

Pfister and Scher $^{20}$ studied the photoconductivity of a-As ${ }_{2} \mathrm{Se}_{3}$. When a pulse light is irradiated to the surface of the thin film of the amorphous semiconductor, the photoelectric current is induced in it. They found that the time dependence of the photoelectric current is expressed by a hyperbolic function. Further the hyperbolic exponent $\alpha$ was found to be $\alpha=T / T_{\mathrm{r}}$, where $T_{\mathrm{r}}$ is shown in eq 23 . It was concluded that the induced electron and positive hole move in accordance with the fractal time stochastic random process. Here the fractal time stochastic random process refers to the phenomenon that the primary process occurs with interval times obeying the distribution function like eq 28 .

It is well-known that the rate of the enzymatic reaction is in the time region from $10^{6} / \mathrm{s}$ to $10^{3} / \mathrm{s}$. Since the enzymatic reaction is due to the local conformational change around the active site on the enzyme, it had been believed that the mean rate of local motion of the enzyme chain is in the same order of the rate of the enzymatic reaction. Go, Noguti, and Nishikawa ${ }^{31}$ studied the local motion of bovine pancreatic trypsin inhibitor (BPTI) by the nomal mode analysis. They found that the slowest rate of the conformational change in BPTI chain occurs in the region about $10^{11} / \mathrm{s}$. In other words, the enzymatic reaction is momentarily completed in picosecond. Therefore the mean rate of the enzymatic reaction is due to frequency of collision between enzymes and substrates.

Schlesinger and Montroll ${ }^{24}$ discussed the asymmetric spectrum of dielectric constant of glasses and polymeric systems. They proposed that the frequency-dependent dielectric constant is indebted to the collision between a dipole and defects in a system, and derived $\mathrm{KWW}$ function as a dielectric relaxation function. In their study it was assumed that the number of defects, which obeys the fractal time stochastic process, is much larger than that of dipoles in a system. This assumption is equal to solve eq 31 with a condition $n_{A} \ll n_{B}$. Above mentioned, since the number of $A$ is same order of that of $B$, i.e., $n_{A} \simeq n_{B}$, it is difficult to adopt their thory to our experiments. It is however important that they also pointed out the significancy of the distribution of the interval time between collisions.

\section{Characteristic Activation Energy of Segments on PSAPS Chain}

Reference temperature $T_{\mathrm{r}}$ is related to the hyperbolic exponent $\beta$ as $\beta=\left(T-T_{\mathrm{r}}\right) / T_{\mathrm{r}}$. According to eq 23 , we obtaine

$$
E_{\mathrm{a}}=k_{\mathrm{B}} T_{\mathrm{r}}=k_{\mathrm{B}} \frac{T}{\beta+1}
$$

Table II. Reference temperatures $T_{\mathrm{r}}$

\begin{tabular}{cccc}
\hline & \multicolumn{3}{c}{$T_{\mathrm{r}} / \mathrm{K}$} \\
\cline { 2 - 4 } Temperature $/{ }^{\circ} \mathrm{C}$ & PSAPS3200 & PSAPS2500 & PSAPS730 \\
\cline { 2 - 4 } & 209 & 206 & 208 \\
20.0 & 213 & 213 & 209 \\
30.0 & 212 & 203 & 204 \\
37.5 & 194 & 200 & 205 \\
40.0 & 202 & 189 & 200 \\
42.5 & 197 & 196 & 187 \\
\hline Average & $205 \pm 7$ & $201 \pm 8$ & $202 \pm 7$ \\
\hline
\end{tabular}

Table III. Comparison of apparent activation energy of polystyrene main chain

\begin{tabular}{lclcc}
\hline \multicolumn{1}{c}{ Author } & Technique & \multicolumn{1}{c}{ Solvent } & $E_{a} / \mathrm{kJ} \mathrm{mol}^{-1}$ & Ref. \\
\hline Valeur & $\mathrm{FD}^{\mathrm{a}}$ & Tripropionin & 4.2 & 4 \\
Nomura & $\mathrm{UR}^{\mathrm{b}}$ & Toluene & 11 & 32 \\
Mashimo & $\mathrm{DR}^{\mathrm{c}}$ & Tolnene & 11.8 & 33 \\
& & Ethylbenzene & 12.1 & 33 \\
Matsuo & ${ }^{19} \mathrm{~F} \mathrm{NMR} \mathrm{C}_{6} \mathrm{D}_{6}$ & 18 & 37 \\
Heartley & ${ }^{1} \mathrm{H} \mathrm{NMR}$ & $\mathrm{CCl}_{4}$ & 14 & 38 \\
Gronski & ${ }^{13} \mathrm{C} \mathrm{NMR}$ & Toluene & 10 & 34 \\
Friedrich & $\mathrm{ESR}^{\mathrm{d}}$ & Toluene & 8.46 & 36 \\
Yokotsuka & $\mathrm{FD}$ & Buthyl acetate & 6.3 & 35 \\
& & Cyclohexane & 6.7 & 35 \\
& & Dioxane & 2.9 & 35 \\
Waldow & & Cyclohexanone & 3.3 & 35 \\
& & Cyclohexane & 21 & 39 \\
& & Tripropionin & 11 & 39 \\
Ono & & 2-Butanone & 8 & 39 \\
Horinaka & $\mathrm{FD}$ & Cyclohexanone & $4.2-9.6$ & 30 \\
& $\mathrm{FD}$ & Cyclohexanone & 10 & 40 \\
& & Butyl acetate & 7.5 & 40 \\
& & Ethyl benzene & 5.4 & 40 \\
& & Benzene & 7.1 & 40 \\
\hline
\end{tabular}

${ }^{\mathrm{a}}$ Fluorescence depolarization. ${ }^{\mathrm{b}}$ Ultrasonic relaxation. ${ }^{\mathrm{c}}$ Dielectric relaxation. ${ }^{\mathrm{d}}$ Electron spin relaxation.

Values of $T_{\mathrm{r}}$ shown in Table II are independent of temperature in this experiment. Thus the characteristic activation energy $E_{\mathrm{a}}$ of a polymer segment is caluculated by mean value of $T_{\mathrm{r}}$. The values of $E_{\mathrm{a}}$ for PSAPS 3200 , PSAPS2500, and PSAPS730 are $7.1 \mathrm{~kJ} \mathrm{~mol}^{-1}$, $7.0 \mathrm{~kJ} \mathrm{~mol}^{-1}$, and $7.0 \mathrm{~kJ} \mathrm{~mol}^{-1}$, respectively. These results suggest that the apparent activation energy of segments on PSAPS chain to be independent of chain length.

Apparent activation energies measured about a polystyrene main chain were listed in Table III. The ultrasonic $^{32}$ and dielectric relaxation ${ }^{33}$ methods, which are able to detect relaxation modes of polymer chains as a function of frequency, gave relatively large values of $E_{\mathrm{a}}$ about $10 \sim 12 \mathrm{~kJ} \mathrm{~mol}^{-1}$. $\mathrm{NMR}^{34}$ methods gave also similar values to the ultrasonic and dielectric relaxation methods. On the other hand, values of apparent activation energy obtained by the fluorescence depolariza$\operatorname{tion}^{4,30,35}$ and the electron spin relaxation ${ }^{36}$ methods tend to reach to the range of $4-10 \mathrm{~kJ} \mathrm{~mol}^{-1}$. Thus the characteristic activation energy of $7 \mathrm{~kJ} \mathrm{~mol}^{-1}$ for PSAPS chain obtained in this study is identical to results reported by many authors.

\section{Apparent Activation Energy of Solvent}

The minimum reaction radii $\xi_{0}$ reflects the transla- 
Polymer Chain Dynamics II.

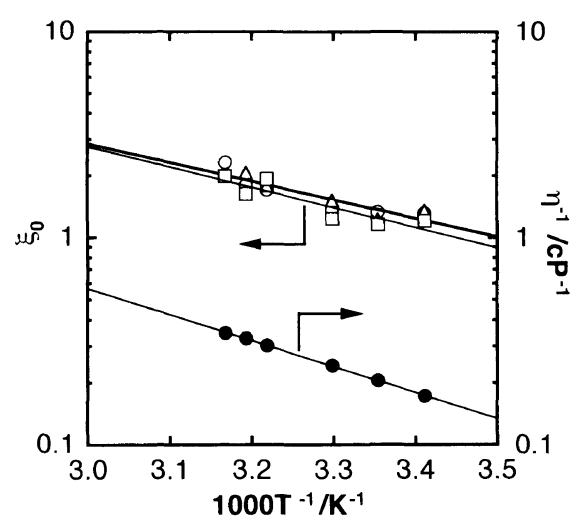

Figure 6. Temperature dependence of minimum reaction radii $\xi_{0}$ and the reciprocal of solvent viscosity $\eta . \square, \triangle$, and $\bigcirc$ indicate the value of $\xi_{0}$ obtained by fluorescence anisotropy decay curves of PSAPS3200, PSAPS2500, and PSAPS730, respectively. denotes the value of $\eta^{-1}$.

tional diffusion coefficient of solvent molecules. $\xi_{0}$ ought to be the same dependence on temperature with the reciprocal of solvent viscosity.

Figure 6 shows the temperature dependence of $\xi_{0}$ obeying the Arrhenius law as

$$
\xi_{0} \sim \exp \left(-\frac{E_{\mathrm{s}}}{k_{\mathrm{B}} T}\right)
$$

where $E_{\mathrm{s}}$ is the apparent activation energy of solvent molecules. The values of $E_{\mathrm{s}}$ for PSAPS3200, PSAPS2500, and PSAPS730 solutions are $6.3 \mathrm{~kJ} \mathrm{~mol}^{-1}, 5.9 \mathrm{~kJ} \mathrm{~mol}^{-1}$, and $5.9 \mathrm{~kJ} \mathrm{~mol}^{-1}$, respectively. Temperature dependence of the reciprocal of solvent viscosity $\eta$, which is measured by an Ubbelohde viscometer, is also shown in Figure 6. The apparent activation energy of solvent molecules was $6.0 \mathrm{~kJ} \mathrm{~mol}^{-1}$. Obviously both $\xi_{0}$ and $\eta^{-1}$ similarly depend on temperature. Therefore it is proved that the influence of the solvent viscosity to orientational relaxation of polymer segments can be estimated by the value of $\xi_{0}$.

\section{CONCLUSION}

We propose the "intermittent process" of polymer segments as one of the probable description for the primary process of orientational relaxation phenomenon of polystyrene back bone. The intermittent process of chain segments is expressed by a hyperbolic function

$$
r(t)=r_{0}\left(t / \tau_{\text {eff }}\right)^{-\beta}
$$

This function was derived on the basis of two ideas. The first is considering orientational relaxation of polymer segments as the reaction between segments and solvent molecules. The second is the distribution of the interval time between relaxation phenomena. That is to say, orientational relaxation of a segment start and stop at intervals.

The hyperbolic exponent $\beta$ is related to the characteristic activation energy $E_{\mathrm{a}}$ of a polymer segment by

$$
E_{\mathrm{a}}=k_{\mathrm{B}} T_{\mathrm{r}}=k_{\mathrm{B}} \frac{T}{\beta+1}
$$

The apparent activation energy of solvent $E_{\mathrm{s}}$ is evaluated by the temperature dependence of minimum reaction radii $\xi_{0}$ as

$$
\xi_{0} \sim \frac{\beta}{r_{0} \tau_{\text {eff }}^{\beta}} .
$$

Hyperbolic relaxation function makes us to estimate separately the characteristics of chain segments itself and the effect of solvent.

\section{REFERENCES}

1. H. Ushiki and F. Tsunomori, Bull. Fac. Gen. Ed. Tokyo Univ. Agric. Technol., 26, 109 (1989).

2. H. Ushiki and K. Ushiki, Bull. Fac. Gen. Ed. Tokyo Univ. Agric. Technol., 27, 105 (1990).

3. H. Ushiki, Rep. Prog. Polym. Phys. Jpn., 35, 419 (1992).

4. B. Valeur and L. Monnerie, J. Polym. Sci., Polym. Phys. Ed., 14, 11 (1976).

5. A. A. Jones and W. H. Stockmayer, J. Polym. Sci., 15, 847 (1977).

6. J. T. Bendler and R. Yaris, Macromolecules, 11, 650 (1978).

7. C. K. Hall and E. Helfand, J. Chem. Phys., 77, 3275 (1982)

8. J. L. Viovy, L. Monnerie, and J. C. Brochon, Macromolecules, 16, 1845 (1983).

9. T. Sasaki, M. Yamamoto, and Y. Nishijima, Macromolecules, 21, 610 (1988)

10. M. D. Ediger, Ann. Rev. Phys. Chem., 42, 225 (1991).

11. B. Valeur, J. P. Jarry, F. Geny, and L. Monnerie, J. Polym. Sci. Polym. Phys. Ed., 13, 667 (1975).

12. B. Valeur, L. Monnerie, and J. P. Jarry, J. Polym. Sci., Polym. Phys. Ed., 13, 675 (1975).

13. H. Takayasu, "Fractal Kagaku," Asakura, Tokyo, 1987.

14. H. Scher, M. F. Schlesinger, and J. T. Bendler, Parity, 6(9), 14 (1991).

15. R. Kohlraush, "Annalen der Physik und Chemie," Poggendorf, 1854 .

16. G. Williams and D. C. Watts, Trans. Faraday Soc., 66, 80 (1971).

17. J. Orenstein, M. Kastner, and D. Monroe, J. Non-Cryst. Solid, 35, 951 (1980)

18. G. Adam and J. H. Gibbs, J. Chem. Phys., 43, 139 (1965).

19. K. L. Ngai, R. W. Rendell, and D. J. Plazek, J. Chem. Phys., 94, 3018 (1991)

20. G. Pfister and H. Scher, Phys. Rev. B, 15, 2062 (1977).

21. J. Tuac, Festkörperprobleme, 22, 85 (1982).

22. K. L. Ngai and K. Murayama, Physica B +C, 117/118, 980 (1983).

23. R. Saito and K. Murayama, Solid State Commun., 63, 625 (1987).

24. M. F. Schlesinger and E. W. Montroll, Proc. Natl. Acad. Sci. U.S.A., 81, 1280 (1984).

25. K. Murayama and T. Ninomiya, Solid State Commun., 53, 125 (1985).

26. F. Tsunomori and H. Ushiki, Polym. J., 28, 576 (1996)

27. K. Horie, K. Tomonume, and I. Mita, Polym. J., 11, 539 (1979).

28. P. Wahl, Biophys. Chem., 10, 91 (1979).

29. T. Nakagawa and Y. Oyanagi, "Saishou Nijyou-hou Niyoru Jikken-Data Kaiseki," Tokyo Daigaku Syuppan-kai, Tokyo, 1982.

30. K. Ono, Y. Okada, S. Yokotsuka, S. Ito, and M. Yamamoto, Polym. J., 26, 199 (1994)

31. N. Go, T. Noguti, and T. Nishikawa, Proc. Natl. Acad. Sci. U.S.A., 80, 3696 (1983).

32. H. Nomura, S. Kato, and Y. Miyahara, Mem. Fac. Eng, Nagoya Univ., 27, 72 (1975).

33. S. Mashimo, Macromolecules, 9, 91 (1976).

34. W. Gronski, T. Schäfer, and R. Peter, Polym. Bull., 1, 319 (1979).

35. S. Yokotsuka, Y. Okada, Y. Tojo, T. Sasaki, and M. Yamamoto, Polym. J., 23, 95 (1991).

36. C. Friedrich, F. Lauprêtre, C. Noël, and L. Monnerie, Macromolecules, 14, 1119 (1981).

37. K. Matsuo, K. F. Kuhlmann, W. H. Yang, F. Geny, and W. H Stockmayer, J. Polym. Sci., Polym. Phys. Ed., 15, 1347 (1977).

38. F. Heartley and B. Wood, Polymer, 19, 1405 (1978).

39. D. A. Waldow, M. D. Ediger, Y. Yamaguchi, Y. Matsushita, and I. Noda, Macromolecules, 24, 3147 (1991).

40. J. Horinaka, S. Amano, S. Ito, and M. Yamamoto, Polym. Prepr Jpn., 44, 3543 (1995). 